\title{
Prior Endovascular Intervention is Not Detrimental to Pedal Bypasses for Ischemic Wounds
}

\author{
Abhisekh Mohapatra, MDa, Mikayla N. Lowenkamp, BS ${ }^{a}$, Jon C. Henry, MDa ${ }^{a}$ Aureline \\ Boitet, MD ${ }^{\mathrm{a}}$, Efthimios D. Avgerinos, MD ${ }^{\mathrm{a}}$, Rabih A. Chaer, MD ${ }^{\mathrm{a}}$, Michel S. Makaroun, MD ${ }^{\mathrm{a}}$, \\ Steven A. Leers, MD $^{\mathrm{a}}$, and Eric S. Hager, $\mathbf{M D}^{\mathrm{a}}$ \\ aDivision of Vascular Surgery, Heart \& Vascular Institute, University of Pittsburgh Medical Center, \\ Pittsburgh, PA, USA
}

\section{Abstract}

\begin{abstract}
Introduction-Endovascular strategies are often preferred for revascularization of ischemic foot wounds secondary to infrapopliteal disease due to the less invasive technique and faster recovery. Bypass is typically reserved for failures or lesions not amenable to balloon angioplasty. However, the effects of an endovascular-first approach on subsequent bypass grafts is largely unknown. This study evaluates the effects of prior tibial interventions on successive bypasses to pedal targets.
\end{abstract}

Methods-Patients who presented with ischemic tissue loss and tibial arterial occlusive disease to UPMC between 2006 and 2013 and underwent a surgical bypass to pedal arteries were included in this study. A retrospective chart review was conducted to obtain patient demographics, past medical history, extent of disease, prior tibial endovascular interventions, the treatment intervention, subsequent interventions, wound healing status, limb salvage, and patient survival. The primary outcome was primary patency of the pedal bypass graft.

Results-From 122 eligible patients, 27 had a prior endovascular tibial intervention (PTI) while 95 had no prior tibial intervention (nPTI) in the treatment of ischemic pedal wounds with mean follow-up of 24.5 and 20.5 months respectively $(\mathrm{P}=0.36)$. The two groups were largely similar in terms of demographics, co-morbidities, wound size, and degree of ischemia. Runoff scores between the two groups were also comparable $(5.0 \pm 1.6$ for PTI and $4.8 \pm 1.9$ for $\mathrm{nPTI}, \mathrm{P}=0.59)$. The plantar artery was a more common target vessel in the PTI group, while the posterior tibial artery was targeted more often in the $\mathrm{nPTI}$ group $(\mathrm{P}=0.04)$. At 12 months, those with a prior tibial intervention exhibited a shorter primary patency $(34.8 \%$ vs. $60.2 \%, \mathrm{P}=0.04)$. In a multivariate model, PTI was a significant risk factor for primary patency loss (HR 2.51, P $=0.004)$. Primaryassisted patency and secondary patency were similar between the two groups. Wound healing was improved in those patients who had a prior endovascular intervention with $63.8 \%$ healed at one

Corresponding Author: Abhisekh Mohapatra MD, Heart \& Vascular Institute, Division of Vascular Surgery, University of Pittsburgh Medical Center, 200 Lothrop St, Suite A-1017, Pittsburgh, PA 15213, USA, 412-802-3333 (Phone), 412-291-1669 (Fax), mohapatraa@upmc.edu.

Publisher's Disclaimer: This is a PDF file of an unedited manuscript that has been accepted for publication. As a service to our customers we are providing this early version of the manuscript. The manuscript will undergo copyediting, typesetting, and review of the resulting proof before it is published in its final citable form. Please note that during the production process errors may be discovered which could affect the content, and all legal disclaimers that apply to the journal pertain. 
year compared to only $34.8 \%$ of those without intervention $(\mathrm{P}=0.01)$. Amputation-free survival was similar $(\mathrm{P}=0.68)$, as was survival alone $(\mathrm{P}=0.50)$.

Discussion-Despite a decrease in primary patency, pedal bypass was not otherwise negatively affected by a prior tibial intervention. Similar primary assisted patency, secondary patency, wound healing, and survival between the two patient populations indicate an endovascular-first approach is a feasible treatment strategy to achieve similar clinical outcomes in the management of ischemic foot wounds.

\section{Introduction}

Critical limb ischemia (CLI), the end stage of peripheral arterial disease (PAD), is a complex disease state characterized by poorer wound healing, increased risk of amputation, and increased mortality [1]. With mortality rates as high as $25 \%$ and amputation rates as high as $40 \%$ [2], early and effective revascularization procedures are critical. This is particularly challenging for CLI with tibial vessel involvement as both surgical and endovascular therapies are associated with poorer limb prognosis compared to more proximal disease [3]. The immediate goal of treatment in patients with CLI is wound healing and limb salvage, which historically has been accomplished through surgical bypass [4].

Improvements in endovascular technologies have provided an alternative treatment strategy that is less invasive yet maintains clinical effectiveness in terms of amputation and survival when compared to bypass surgery [5]. Specifically, endovascular interventions for infrapopliteal arterial disease have been shown to be equivalent to distal bypass in limb salvage and amputation-free survival, though patency rates are typically poorer [6, 7]. In cases where initial endovascular approaches fail, open surgical bypass is often successful in restoring adequate perfusion for limb salvage [8]. The effects of prior endovascular interventions on subsequent bypasses at the infra-popliteal level have not been well described. Therefore, this study's objective is to determine whether an endovascular-first approach has deleterious effects on the success of a subsequent inframalleolar bypass for ischemic wounds.

\section{Material and Methods}

\subsection{Study Approval}

This study was approved by the University of Pittsburgh Medical Center (UPMC) Institutional Review board. Patient consent was deemed unnecessary as the study constitutes a retrospective chart review.

\subsection{Inclusion/Exclusion Criteria}

The population included in this study were patients who presented to UPMC between 2006 and 2013 with ischemic tissue loss and tibial arterial occlusive disease and underwent a bypass to the pedal arteries (dorsalis pedis, distal posterior tibial, or plantar arteries). Eligible patients were required to have at least one follow-up appointment with documentation of the healing status of their wound. Patients were excluded if the index procedure was performed at an outside institution or if the wound on the treated limb could not be characterized or confirmed. Additionally, patients were also excluded if their wound 
extended above the ankle or the affected limb had been treated with a prior bypass distal to the popliteal artery.

\subsection{Data Collection}

Eligible study participants were identified from a prospectively maintained list of patients who have undergone lower extremity bypasses. Additional patients were acquired by a search of the electronic medical record (EMR). All patients in the study had tibial occlusive disease; patients with concomitant disease more proximally were also included. Patients were dichotomized based upon whether they had undergone a prior tibial intervention (PTI) or no prior tibial intervention (nPTI) before the index bypass operation was performed.

Pertinent patient characteristics of those who met the inclusion criteria were obtained through a retrospective review of the EMR. These characteristics included patient demographics, past medical history, smoking history, and prior medications. Diagnoses of interest in the past medical history included coronary artery disease, heart failure, hypertension, hyperlipidemia, diabetes mellitus, hypercoagulable states, chronic obstructive pulmonary disease, lower extremity neuropathy, and cerebrovascular disease. Using the medical record, wounds were retrospectively categorized based on Wound, Ischemia, foot Infection (WIfI) criteria, which have been validated as a predictor of amputation risk and perceived benefit of revascularization [9]. Treatment characteristics such as timing, number of procedures, and initial complications were all considered. Distal outflow resistance was assessed by runoff scores as previously described in Society for Vascular Surgery reporting standards [10].

The primary outcome was primary graft patency, defined as freedom from any stenosis or intervention, as documented by subsequent inpatient or outpatient visits. Primary assisted and secondary patency, wound healing, major (above-ankle) amputation, major adverse limb events (MALE, defined as a composite of major amputation or major reintervention) [11], and mortality were studied as secondary outcomes.

\subsection{Statistical Analysis}

Data was compiled in Excel (Microsoft, Redmond, WA) and analyzed using Stata 15.0 (StataCorp, College Station, TX). Baseline characteristics were compared using Student's Ttest or the Chi-squared test for continuous and categorical data, respectively. 30-day postoperative outcomes were compared in a similar fashion. Time-dependent outcomes (wound healing, amputation, mortality, and patency outcomes) were compared by survival analysis. Kaplan-Meier curves, stratified by treatment group, were used to depict differences, and the log-rank test was used to assess them. Cox proportional hazards models were utilized for identification of univariate and multivariate risk factors for the primary outcome. The final multivariate model was selected in a backward-stepwise fashion starting with significant or near-significant univariate risk factors. For all statistical tests, a P-value of $<0.05$ was considered to be statistically significant. For all Kaplan-Meier graphs, standard error was $₫ 10 \%$ at all points on all curves shown. 


\section{Results}

\subsection{Patient Population}

We identified 130 patients who underwent pedal bypass during the study period. Of these, eight patients were excluded for having undergone a prior distal bypass. The remaining 122 patients met the eligibility criteria to participate in this study; 27 of these patients had a prior endovascular tibial intervention (PTI) while 95 did not (nPTI). The mean follow-up time was 20.5 months for the PTI group and 24.5 months for the nPTI group $(\mathrm{P}=0.36)$.

\subsection{Baseline Characteristics and Procedural Details}

Demographic comparisons showed no statistically significant difference in age, gender or race between the PTI and nPTI groups (Table I). Additionally, past medical history was similar between the two groups except that PTI patients were more likely to be on warfarin therapy $(37.0 \%$ vs. $16.8 \%, \mathrm{P}=0.05)$ and antiplatelet therapy $(74.1 \%$ vs. $42.1 \%, \mathrm{P}=0.03)$.

Wound size and degree of ischemia were similar between the two comparison groups (Table II). No patients demonstrated systemic infections prior to the bypass procedure, but PTI patients were more likely to have moderate localized cellulitis or purulence pre-operatively ( $44.4 \%$ vs. $20.0 \%, \mathrm{P}=0.03$ ). Runoff scores between the two patient groups were similar, indicating comparable pedal outflow resistance for the bypasses (Table II).

The most common inflow artery was the below knee popliteal artery (70\%) and the frequency of use was similar between groups. In contrast, there was a statistically significant difference in target vessels between groups. More plantar artery targets were chosen as target vessels in the PTI group (25.9\% vs. 9.5\%) and more posterior tibial artery targets in the nPTI group $(14.7 \%$ vs $3.7 \%, \mathrm{P}=0.04)$. The type of conduit was similar in both groups (Table III).

\subsection{Wound Care}

The wound care regimen was the same in all patients regardless of treatment performed. Wound care was coordinated in all patients by the vascular surgeon, who would evaluate wounds in the office at least on a biweekly basis and determine the optimal local wound care strategy alone or in conjunction with a podiatrist. Dressing changes and other aspects of wound care may have been provided by the patient or family members, visiting nurses, or staff at a skilled nursing facility as appropriate for each patient's individual needs.

\subsection{Post-Operative Outcomes}

30-day post-operative outcomes are detailed in Table IV and were generally similar. Mortality rates were low and similar between the PTI and nPTI groups ( $0 \%$ vs. $1.1 \%, \mathrm{P}=$ $1.00)$, as were major amputation rates $(0 \%$ vs. $1.1 \%, \mathrm{P}=1.00)$. There was no difference in the re-intervention rate in the immediate post-operative period $(14.8 \%$ vs. $10.5 \%, \mathrm{P}=0.51)$. Hospital length of stay, discharge disposition, and ambulatory status were also similar between groups. 


\subsection{Long-Term Patency and Clinical Outcomes}

Primary patency was significantly lower in the PTI group compared to the $\mathrm{nPTI}$ group $(\mathrm{P}=$ 0.04; Fig. IA). At one year, primary patency was $34.8 \%$ vs. $60.2 \%$ in the PTI and nPTI groups, respectively. Upon Cox regression analysis, PTI was found to be a predictor of loss of primary patency (HR 1.81, 95\% CI 1.01-3.25, P = 0.045). Other significant or nearsignificant univariate predictors for loss of primary patency included CHF (HR 1.79, 95\% CI 0.99-3.21, P = 0.052), history of CVA (HR 2.48, 95\% CI 1.24-4.95, P = 0.01), current smoking (HR 2.12, 95\% CI 1.08-4.13, P = 0.03), suprageniculate inflow artery (HR 1.99, $95 \%$ CI 1.16-3.43, P = 0.01), and non-GSV conduit (HR 1.76, 95\% CI 1.01-3.09, $\mathrm{P}=$ 0.048). A multivariate regression model was constructed using these predictors and is shown in Table V. Adjusting for these factors, prior tibial intervention was a significant risk factor for loss of primary patency (HR 2.51, 95\% CI 1.34-4.72, P = 0.004). Of note, bypass target artery and runoff scores were investigated and did not contribute significantly to our multivariate model $(\mathrm{P}=0.97$ and $\mathrm{P}=0.28$, respectively, by a likelihood ratio test).

Primary assisted $(\mathrm{P}=0.58$; Fig. IB $)$ and secondary patency $(\mathrm{P}=0.47$; Fig. IC) were similar between the two groups. At one year, primary assisted patency was $75.0 \%$ vs. $79.7 \%$ and secondary patency was $75.0 \%$ vs. $80.2 \%$, respectively. PTI was not found to be a univariate risk factor for loss of primary assisted $(\mathrm{P}=0.63)$ or secondary patency $(\mathrm{P}=0.52)$. A multivariate model was constructed for each outcome with the same factors listed in Table $\mathrm{V}$; in these models, PTI remained a non-significant predictor of loss of primary assisted $(\mathrm{P}=$ $0.25)$ or secondary patency $(\mathrm{P}=0.23)$.

Wound healing rates were higher in the PTI group $(\mathrm{P}=0.01$; Fig. II). The rate of successful wound healing was $63.8 \%$ in the PTI group compared to $34.8 \%$ in the nPTI group at one year. Freedom from MALE was similar between groups $(\mathrm{P}=0.77$; Fig. IIIA) and was $79.6 \%$ vs. $77.0 \%$ at one year. Amputation-free survival was also similar $(72.1 \%$ vs. $76.2 \%, \mathrm{P}=$ 0.68 ; Fig. IIIB), as was survival alone (78.3\% vs. $87.6 \%, \mathrm{P}=0.50$; Fig. IIIC).

\section{Discussion}

This study served to determine the effects of prior endovascular interventions on subsequent inframalleolar bypasses with a pedal target for ischemic foot wounds. We found that, in our cohort, prior intervention did not significantly compromise wound healing, limb salvage, or mortality rates in patients with tibial arterial disease. There was, however, a notably decreased primary patency in the patients with a prior intervention. This may be linked to the need for more distal target arteries after a prior intervention. Though our analysis did not identify the target artery as a predictor of failure, we noted that $25.9 \%$ of the PTI patients underwent bypass to a plantar vessel compared to $9.5 \%$ of the nPTI patients. The use of a plantar vessel in lieu of the distal posterior tibial artery may be due to the compromise of the latter by the prior intervention. Studies on femoropoliteal interventions have shown a similar effect in necessitating the use of more distal bypass targets [12]. A more distal bypass often requires a longer, potentially more compromised conduit placed in an area of increased pressure with ambulation. This could in turn impact primary patency. Despite the large difference in primary patency between groups, primary assisted and secondary patency were 
nearly identical, suggesting that a prior tibial intervention does not significantly impact the overall patency of a pedal bypass, albeit with a greater need for re-interventions.

A second explanation for our findings is that those who underwent prior tibial intervention had more significant, multi-level disease. However, we would expect that, based on our general practice, a heavier disease burden would predispose a patient to undergo a primary bypass operation rather than an endovascular intervention. Furthermore, a number of our findings indicate that our results are not a consequence of disease burden alone. We found no difference in the inflow artery used for the bypass, suggesting the degree of more proximal disease was similar. While a suprageniculate inflow was found to be a risk factor for primary patency loss, PTI remained a risk factor after adjusting for the inflow effect. Runoff scores, a measure of the distal outflow, were similar between groups and not found to be a predictor in our model.

The rate of wound healing was significantly faster in the patients with PTI. While the reason for this observation is unclear, one would expect that the prior tibial angioplasty may have begun the wound healing process, resulting in a lower wound burden in these patients at the time of their bypass operation. The WIfI wound scores, however, were similar between the two groups. Another potential explanation for this difference in healing rates is that the PTI patients may have been receiving treatment for longer periods of time prior to their bypass procedure, thereby optimizing their co-morbidities to improve overall healing. Diabetes mellitus, renal impairment, and poor nutritional status are all known to impair wound healing and may have been better optimized in the PTI group at the time of the bypass [13]. Nevertheless, prior tibial intervention does not appear to have a detrimental effect on wound healing.

Aside from wound healing, we found no difference in the rate of any other clinical limbrelated outcome. The similarities in major adverse limb events and amputation-free survival indicate that these patients, and the bypass grafts themselves, seem to minimally affected by a prior tibial intervention. Our findings suggest that an endovascular-first approach is reasonable in patients with tissue loss and tibial artery occlusive disease and does not significantly impact the clinical success rate of a subsequent bypass operation.

The limitations of this study arise from its retrospective nature. There was likely inherent selection bias; in particular, patients not offered a prior endovascular intervention may have undergone a primary bypass operation due to a greater anatomic disease burden or a more severe wound. There may also have been some patients who no longer had suitable anatomy for a bypass after having a prior tibial intervention; these patients would not be included in our study. Additionally, the medical records detailing wound characteristics and size were largely non-uniform, often providing somewhat subjective documentation that made it more difficult to score wounds by WIfI criteria. Inconsistent patient follow-up also made it difficult to document exact time of wound healing. Finally, pedal bypass is a relatively infrequent procedure and a larger sample size would have allowed for more precise estimates and identification of risk factors for bypass failure after a prior intervention. 
Despite these limitations, our study demonstrates that the secondary patency, wound healing, and limb salvage rates after a pedal bypass are not negatively affected by a prior endovascular tibial intervention. In many patients, an endovascular-first approach may be beneficial and does not preclude success with a subsequent distal bypass operation.

\section{Conclusion}

In patients with infrapopliteal arterial occlusive disease and ischemic foot wounds, endovascular tibial intervention is commonly used as a first-line treatment as it is less invasive and better tolerated than surgical bypass. This study demonstrates that, despite a decreased primary patency attributable to the need for more distal target vessels, prior endovascular tibial interventions do not significantly impact primary assisted or secondary patency, wound healing, or amputation rates in patients who underwent a subsequent inframalleolar bypass procedure. Endovascular tibial intervention is a lower-risk, reasonable treatment choice in these patients and only marginally affects the outcome of a subsequent pedal bypass.

\section{Acknowledgments}

Funding: No funding was received for the completion of this study.

\section{References}

1. Abu Dabrh AM, Steffen MW, Undavalli C, et al. The natural history of untreated severe or critical limb ischemia. J Vasc Surg. 2015; 62(6):1642-51.e3. [PubMed: 26391460]

2. Armstrong EJ, Bishu K, Waldo SW. Endovascular Treatment of Infrapopliteal Peripheral Artery Disease. Curr Cardiol Rep. 2016; 18(4):34. [PubMed: 26915012]

3. Chen Q, Shi Y, Wang Y, et al. Patterns of disease distribution of lower extremity peripheral arterial disease. Angiology. 2015; 66(3):211-8. [PubMed: 24650949]

4. Huang ZS, Schneider DB. Endovascular intervention for tibial artery occlusive disease in patients with critical limb ischemia. Semin Vasc Surg. 2014; 27(1):38-58. [PubMed: 25812758]

5. Fu X, Zhang Z, Liang K, et al. Angioplasty versus bypass surgery in patients with critical limb ischemia-a meta-analysis. Int J Clin Exp Med. 2015; 8(7):10595-602. [PubMed: 26379849]

6. Soderstrom MI, Arvela EM, Korhonen M, et al. Infrapopliteal percutaneous transluminal angioplasty versus bypass surgery as first-line strategies in critical leg ischemia: a propensity score analysis. Ann Surg. 2010; 252(5):765-73. [PubMed: 21037432]

7. European Stroke O. Tendera M, Aboyans V, et al. ESC Guidelines on the diagnosis and treatment of peripheral artery diseases: Document covering atherosclerotic disease of extracranial carotid and vertebral, mesenteric, renal, upper and lower extremity arteries: the Task Force on the Diagnosis and Treatment of Peripheral Artery Diseases of the European Society of Cardiology (ESC). Eur Heart J. 2011; 32(22):2851-906. [PubMed: 21873417]

8. Conte MS. Critical appraisal of surgical revascularization for critical limb ischemia. J Vasc Surg. 2013; 57(2 Suppl):8S-13S. [PubMed: 23336860]

9. Mills JL Sr, Conte MS, Armstrong DG, et al. The Society for Vascular Surgery Lower Extremity Threatened Limb Classification System: risk stratification based on wound, ischemia, and foot infection (WIfI). J Vasc Surg. 2014; 59(1):220-34. e1-2. [PubMed: 24126108]

10. Rutherford RB, Baker JD, Ernst C, Johnston KW, et al. Recommended standards for reports dealing with lower extremity ischemia: revised version. J Vasc Surg. 1997; 26(3):517-38. [PubMed: 9308598] 
11. Conte MS, Geraghty PJ, Bradbury AW, et al. Suggested objective performance goals and clinical trial design for evaluating catheter-based treatment of critical limb ischemia. J Vasc Surg. 2009; 50(6):1462-73. e1-3. [PubMed: 19897335]

12. Gifford SM, Fleming MD, Mendes BC, et al. Impact of femoropopliteal endovascular interventions on subsequent open bypass. J Vasc Surg. 2016; 64(3):623-8. [PubMed: 27288105]

13. Stadelmann WK, Digenis AG, Tobin GR. Impediments to wound healing. Am J Surg. 1998; 176(2A Suppl):39S-47S. 


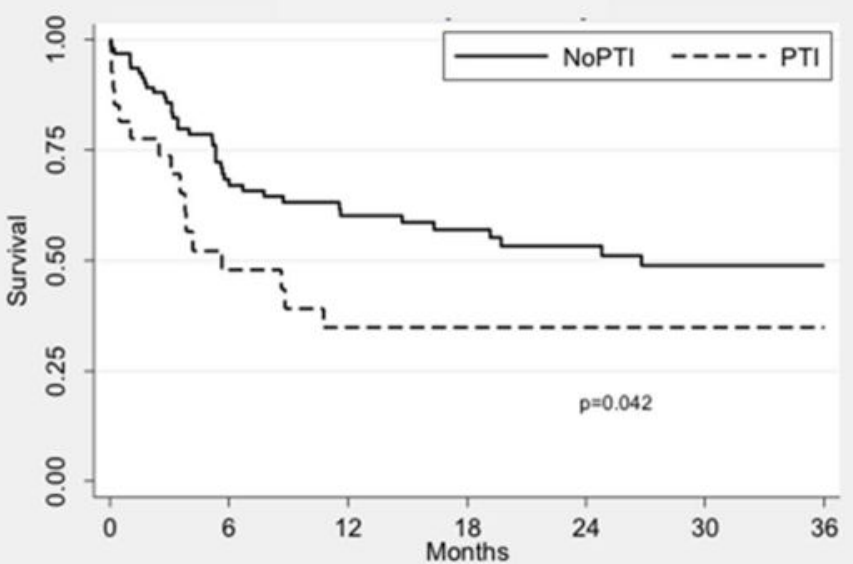

Number at risk

$\begin{array}{lllccccc}\text { NoPTI } & 95 & 53 & 40 & 34 & 25 & 17 & 16 \\ \text { PTI } & 27 & 11 & 8 & 7 & 5 & 4 & 4\end{array}$

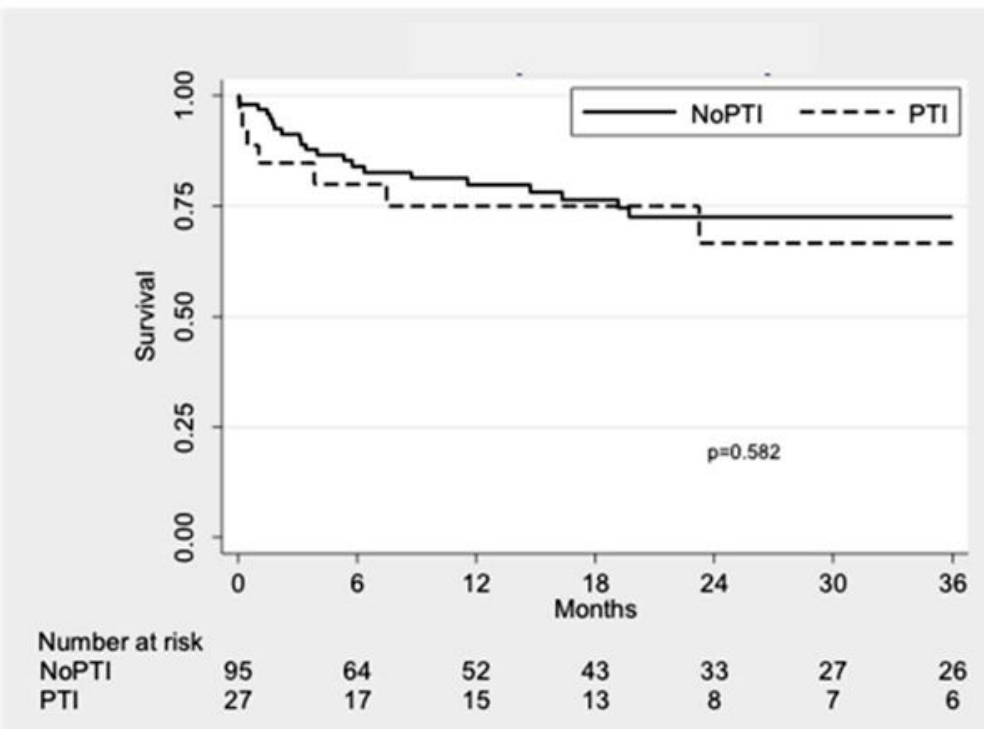

Ann Vasc Surg. Author manuscript; available in PMC 2019 July 01. 


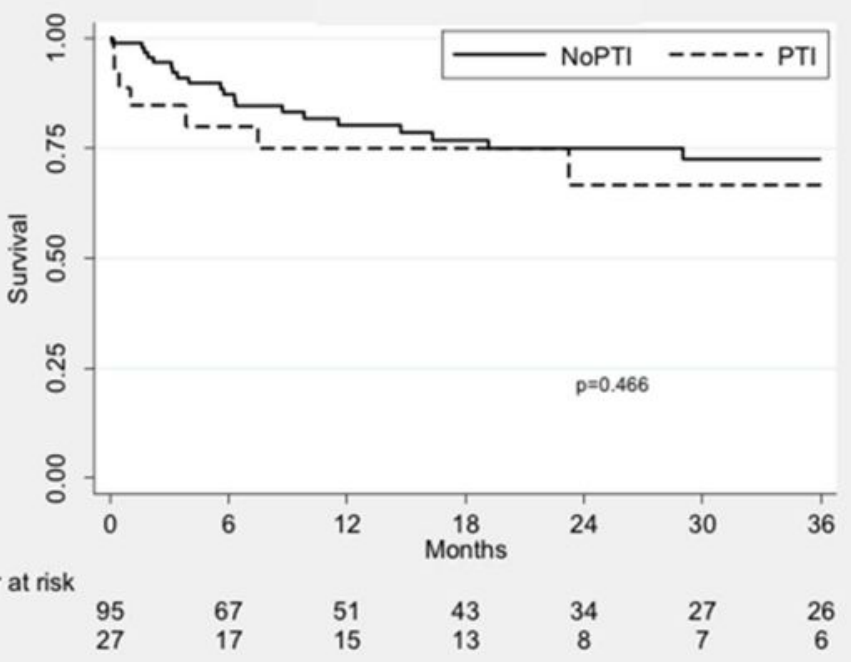

Fig. 1.

Kaplan-Meier curves showing patency outcomes stratified by treatment group. The three curves show primary (A), primary assisted (B), and secondary patency (C). PTI: prior tibial intervention; NoPTI: no prior tibial intervention. 


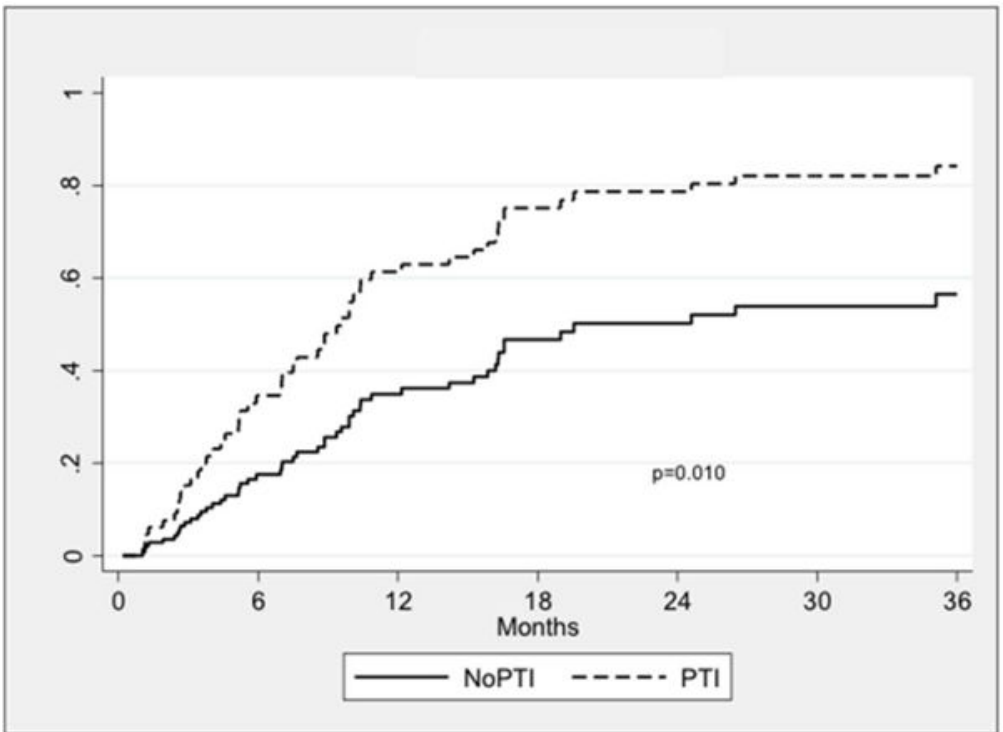

Fig. 2.

Kaplan-Meier curves showing wound healing rates stratified by treatment group. PTI: prior tibial intervention; NoPTI: no prior tibial intervention. 

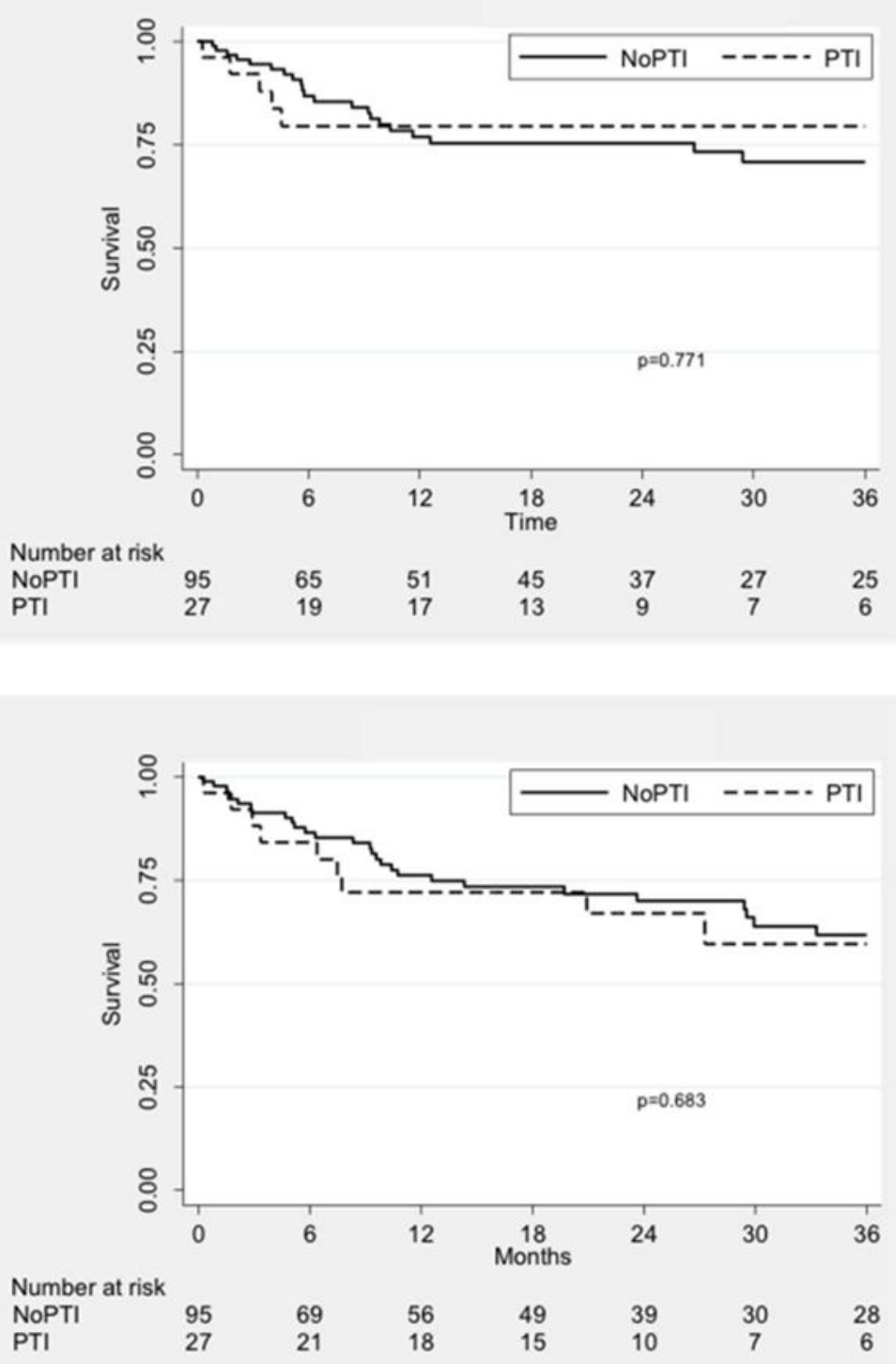

Ann Vasc Surg. Author manuscript; available in PMC 2019 July 01. 


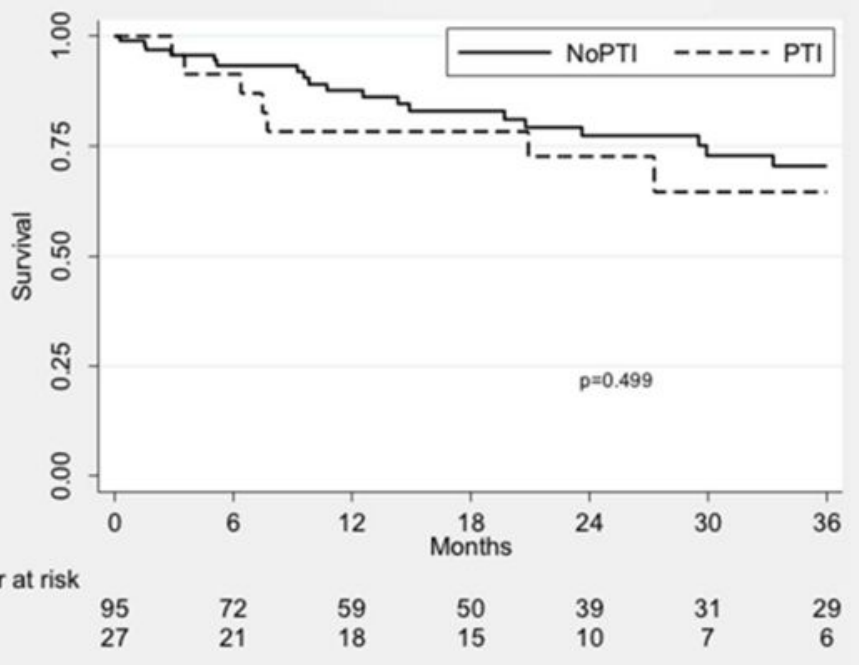

Fig. 3.

Kaplan-Meier curves showing freedom from MALE (A), amputation-free survival (B), and overall survival (C), stratified by treatment group. PTI: prior tibial intervention; NoPTI: no prior tibial intervention. 


\section{Table I}

Baseline characteristics, stratified by treatment group.

\begin{tabular}{|lccc|}
\hline Factor & $\begin{array}{c}\text { PTI } \\
\text { N=27 }\end{array}$ & $\begin{array}{c}\text { No PTI } \\
\text { N=95 }\end{array}$ & P-Value \\
\hline & Demographics & & \\
\hline Age (mean) & $72.0 \pm 9.9$ & $69.8 \pm 9.8$ & 0.425 \\
Gender (\% Male) & $22(81.5 \%)$ & $72(75.8 \%)$ & 0.613 \\
Ethnicity (\% Minority) & $5(18.5 \%)$ & $19(20.0 \%)$ & 1.000 \\
\hline & Medical History & & \\
\hline Diabetes mellitus & $22(81.5 \%)$ & $79(83.2 \%)$ & 0.781 \\
Renal Insufficiency & $8(29.6 \%)$ & $37(38.9 \%)$ & 0.499 \\
End-Stage Renal Disease & $4(14.8 \%)$ & $22(23.2 \%)$ & 0.433 \\
Hypertension & $21(77.8 \%)$ & $83(87.4 \%)$ & 0.351 \\
Hyperlipidemia & $17(63.0 \%)$ & $47(49.5 \%)$ & 0.308 \\
Prior Myocardial Infarction & $8(29.6 \%)$ & $32(33.7 \%)$ & 0.870 \\
Coronary Artery Disease & $19(70.4 \%)$ & $63(66.3 \%)$ & 0.870 \\
Coronary Artery Bypass & $5(18.5 \%)$ & $30(31.6 \%)$ & 0.232 \\
Chronic Obstructive Pulm. Dis. & $5(18.5 \%)$ & $7(7.4 \%)$ & 0.135 \\
Cerebral Vascular Accident & $4(14.8 \%)$ & $12(12.6 \%)$ & 0.752 \\
Tobacco Smoking History & $14(51.9 \%)$ & $45(47.4 \%)$ & 0.828 \\
Warfarin Therapy & $\mathbf{1 0 ( 3 7 . 0 \% )}$ & $\mathbf{1 6 ( 1 6 . 8 ) \%}$ & $\mathbf{0 . 0 4 6}$ \\
Statin Therapy & $11(40.7 \%)$ & $48(50.5 \%)$ & 0.497 \\
B-Blocker Therapy & $14(51.9 \%)$ & $59(62.1 \%)$ & 0.461 \\
Anti-Platelet Therapy & $\mathbf{2 0 ( 7 4 . 1 \% )}$ & $\mathbf{4 0 ( 4 2 . 1 \% )}$ & $\mathbf{0 . 0 2 5}$ \\
\hline
\end{tabular}

PTI: prior tibial intervention; nPTI: no prior tibial intervention. 


\section{Table II}

Pre-operative wound characteristics scored by Wound, Ischemia, foot Infection (WIfI) classification system.

\begin{tabular}{|c|c|c|c|}
\hline Factor & $\begin{array}{c}\text { PTI } \\
\text { N=27 }\end{array}$ & $\begin{array}{l}\text { No PTI } \\
\mathbf{N}=95\end{array}$ & P-Value \\
\hline \multicolumn{4}{|c|}{ Wound Status } \\
\hline No & 0 & 0 & \multirow{4}{*}{0.759} \\
\hline Minor & $5(18.5 \%)$ & $15(15.8 \%)$ & \\
\hline Major & $18(66.7 \%)$ & $60(63.2 \%)$ & \\
\hline Extensive & $4(14.8 \%)$ & $20(21.1 \%)$ & \\
\hline \multicolumn{4}{|c|}{ Ischemia } \\
\hline Grade 0 (ABI $\geq 0.8, \mathrm{TP} \geq 60$ ) & $1(3.7 \%)$ & $8(8.4 \%)$ & \multirow{4}{*}{0.254} \\
\hline Grade 1 (ABI 0.6-0.79, TP 40-59) & $7(25.9 \%)$ & $11(11.6 \%)$ & \\
\hline Grade 2 (ABI 0.4-0.59, TP 30-39) & $1(3.7 \%)$ & $9(9.5 \%)$ & \\
\hline Grade $3(\mathrm{ABI}<0.4, \mathrm{TP}<30$ & $11(40.7 \%)$ & $35(36.8 \%)$ & \\
\hline \multicolumn{4}{|c|}{ Existing Foot Infection } \\
\hline No & $10(37.0 \%)$ & $55(57.9 \%)$ & \multirow{4}{*}{0.034} \\
\hline Mild ( $<2 \mathrm{~cm}$ Cellulitis) & $5(18.5 \%)$ & $21(22.1 \%)$ & \\
\hline Moderate (Cellulitis or Purulence) & $12(44.4 \%)$ & $19(20.0 \%)$ & \\
\hline Extensive (Sepsis) & $\mathbf{0}$ & $\mathbf{0}$ & \\
\hline \multicolumn{4}{|c|}{ Target Status } \\
\hline Runoff Score & $5.0 \pm 1.6$ & $4.8 \pm 1.9$ & 0.594 \\
\hline
\end{tabular}

PTI: prior tibial intervention; nPTI: no prior tibial intervention. ABI: ankle-brachial index; TP: toe pressure. 
Table III

Technical details of bypass operations.

\begin{tabular}{|lccc|}
\hline Factor & $\begin{array}{c}\text { PTI } \\
\text { N=27 }\end{array}$ & $\begin{array}{c}\text { No PTI } \\
\text { N=95 }\end{array}$ & P-Value \\
\hline \multicolumn{4}{c}{ Inflow Artery } \\
Above Knee & $8(29.7 \%)$ & $25(26.3 \%)$ & 0.923 \\
Below Knee & $19(70.3 \%)$ & $70(73.7 \%)$ & \\
\hline \multicolumn{4}{c}{ Target Artery } \\
\hline Dorsalis Pedal Artery & $\mathbf{1 9}(\mathbf{7 0 . 3 \%})$ & $\mathbf{7 2}(\mathbf{7 5 . 8 \% )}$ & \\
Posterior Tibial Artery & $\mathbf{1 ( 3 . 7 \% )}$ & $\mathbf{1 4}(\mathbf{1 4 . 7 \%})$ & $\mathbf{0 . 0 3 9}$ \\
Plantar Artery & $\mathbf{7 ( 2 5 . 9 \% )}$ & $\mathbf{9 ( 9 . 5 \% )}$ & \\
\hline & Conduit & & \\
\hline Great Saphenous Vein & $24(88.9 \%)$ & $71(74.7 \%)$ & \\
Small Saphenous Vein & $1(3.7 \%)$ & $3(3.2 \%)$ & \\
Arm Vein & 0 & $4(4.2 \%)$ & 0.517 \\
Spiced Vein & $2(7.4 \%)$ & $16(16.8 \%)$ & \\
Prosthetic Graft & 0 & $1(1.1 \%)$ & \\
\hline
\end{tabular}

PTI: prior tibial intervention; nPTI: no prior tibial intervention. 


\section{Table IV}

30-day post-operative outcomes.

\begin{tabular}{|lccc|}
\hline Factor & PTI & No PTI & P-value \\
\hline Length of Stay (mean \pm SD) & $12.7 \pm 6.0$ & $10.2 \pm 4.2$ & 0.179 \\
\# Ambulatory & $18(66.7 \%)$ & $74(77.9 \%)$ & 0.346 \\
\# Discharge Home & $13(48.1 \%)$ & $33(34.7 \%)$ & 0.297 \\
\hline & 30-Day Complications & & \\
\hline Cardiac or Respiratory Arrest & $1(3.7 \%)$ & $4(4.2 \%)$ & 1.000 \\
Death & 0 & $1(1.1 \%)$ & 1.000 \\
Urinary Tract Infection & $1(3.7 \%)$ & $10(10.5 \%)$ & 0.453 \\
Deep Vein Thrombosis & 0 & 0 & 1.000 \\
Minor Amputations & $6(22.2 \%)$ & $33(34.7 \%)$ & 0.319 \\
Major Amputations & 0 & $1(1.1 \%)$ & 1.000 \\
Myocardial Infarction & $1(3.7 \%)$ & $4(4.2 \%)$ & 1.000 \\
Renal Failure & 0 & $3(3.2 \%)$ & 1.000 \\
Pneumonia & 0 & $2(2.1 \%)$ & 1.000 \\
Cerebral Vascular Accident & 0 & $1(1.1 \%)$ & 1.000 \\
Wound Complication & $2(7.4 \%)$ & $16(16.8 \%)$ & 0.357 \\
Second Procedure & $4(14.8 \%)$ & $10(10.5 \%)$ & 0.509 \\
\hline
\end{tabular}

PTI: prior tibial intervention; nPTI: no prior tibial intervention. 


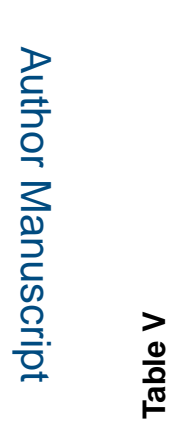

